\title{
The effects of chemical composition on electron delocalization and magnetic ordering in ilvaite, $\mathrm{Ca}\left[\mathrm{Fe}^{2+}, \mathrm{Fe}^{3+}\right]\left[\mathrm{Fe}^{2+}\right] \mathrm{Si}_{2} \mathrm{O}_{7} \mathrm{O}(\mathrm{OH})$
}

\author{
Cherie R. Dotson and B. J. Evans ${ }^{a}$ ) \\ Department of Chemistry, University of Michigan, Ann Arbor, Michigan 48109-1055
}

\begin{abstract}
Substitutional impurities are expected to have significant influences on long-range, order-disorder phenomena associated with magnetic ordering and electron delocalization in mixed-valence solids. These influences have been investigated by means of superconducting quantum interference device susceptometry and ${ }^{57} \mathrm{Fe}$ Mössbauer spectroscopy on more than 40 mineral specimens of the mixed-valence solid ilvaite, $\mathrm{Ca}\left[\mathrm{Fe}^{2+}, \mathrm{Fe}^{3+}\right]\left[\mathrm{Fe}^{2+}\right] \mathrm{Si}_{2} \mathrm{O}_{7} \mathrm{O}(\mathrm{OH})$, with manganese contents between 0.2 and $10 \mathrm{wt} \%$. At $295 \mathrm{~K}$, the ${ }^{57} \mathrm{Fe}$ Mössbauer spectra are quite diverse, exhibiting different numbers of lines, varying degrees of resolution and line broadening. The complexity of the spectra correlate well with increasing Mn content. At $160 \mathrm{~K}$, the spectra are remarkably similar and are characterized by three overlapping subspectra. The Isomer shifts are: $1.09,1.07$, and $0.64 \mathrm{~mm} \mathrm{~s}^{-1}$, respectively, which may be unambiguously assigned to $2 \mathrm{Fe}^{2+}$ and $1 \mathrm{Fe}^{3+}$ species. The magnetic ordering transition near $120 \mathrm{~K}$, associated with the ordering of the moments of the $\mathrm{Fe}^{2+}$ and $\mathrm{Fe}^{3+}$ ions involved in electron delocalization, is not evident in the susceptibility data. Further, the susceptibility versus temperature curves exhibited no qualitative changes with varying Mn content. The magnetic transition associated with the ordering of the magnetic moments of the $\mathrm{Fe}^{2+}$ ion, not involved in electron delocalization, is clearly evident in the susceptibility data and exhibits a systematic dependence on Mn content, occurring at 27, 26, 25, and $23 \mathrm{~K}$ for Mn contents of 0.23 , $0.70,0.90$, and $0.97 \mathrm{wt} \%$. For Mn contents above $1 \mathrm{wt} \%$, this magnetic transition is not observed.

(C) 1999 American Institute of Physics. [S0021-8979(99)53808-5]
\end{abstract}

\section{INTRODUCTION}

Because of the availability of large single crystals with variable compositions, mixed-valence minerals present unique opportunities to investigate the influence of structure and composition on magnetic and electronic order-disorder phenomena. The study of such systems could have important implications relative to the development of novel materials with specialized electronic and magnetic properties.

One such mineral that has received a considerable amount of attention in this regard is the mixed-valence, iron silicate, ilvaite, $\mathrm{Ca}\left[\mathrm{Fe}^{2+}, \mathrm{Fe}^{3+}\right]\left[\mathrm{Fe}^{2+}\right] \mathrm{Si}_{2} \mathrm{O}_{7} \mathrm{O}(\mathrm{OH})$. The structure of ilvaite can be described as ribbons of double rows of edge-shared octahedra containing $\mathrm{Fe}^{2+}$ and $\mathrm{Fe}^{3+}$ in sites designated as $M 11$ and $M 12$, respectively. Above and below the ribbons are octahedral sites, designated as $M 2$, which contain $\mathrm{Fe}^{2+}$ ions. The major substitutional impurity in ilvaite is manganese which has been found to preferentially occupy the $M 2$ site. $^{1}$ The ribbons are held together in a three dimensional framework by $\mathrm{Ca}^{2+}$ ions and $\mathrm{Si}_{2} \mathrm{O}_{7}$ tetrahedral groupings.

Despite the interest in ilvaite, many ambiguities remain concerning the magnetic and electronic properties of this mineral. ${ }^{2-5}$ In addition, there are only a limited number of systematic studies of such properties as a function of chemical composition. Based on previous studies, two magnetic transitions are expected to occur in ilvaite near $\sim 120 \mathrm{~K}^{5-8}$

a)Electronic mail: bjemag@umich.edu
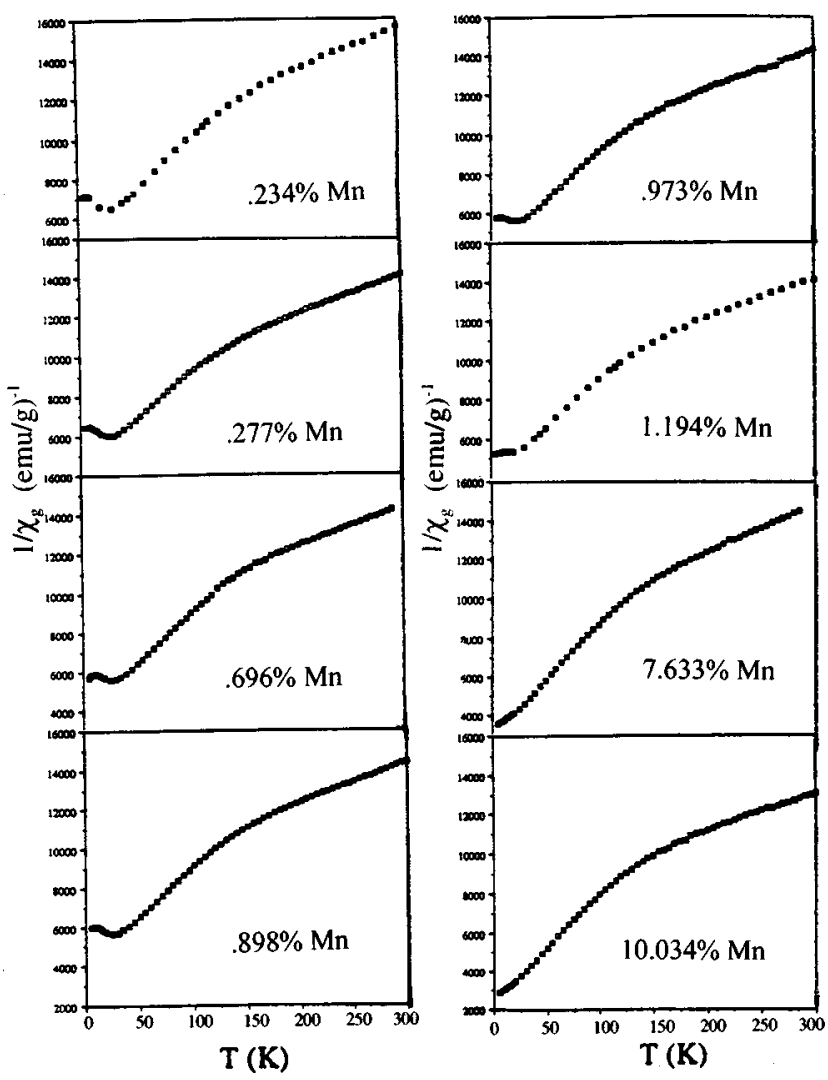

FIG. $1.1 / \chi_{g}$ vs $T$ from 4 to $300 \mathrm{~K}$ for ilvaites with different manganese contents. 


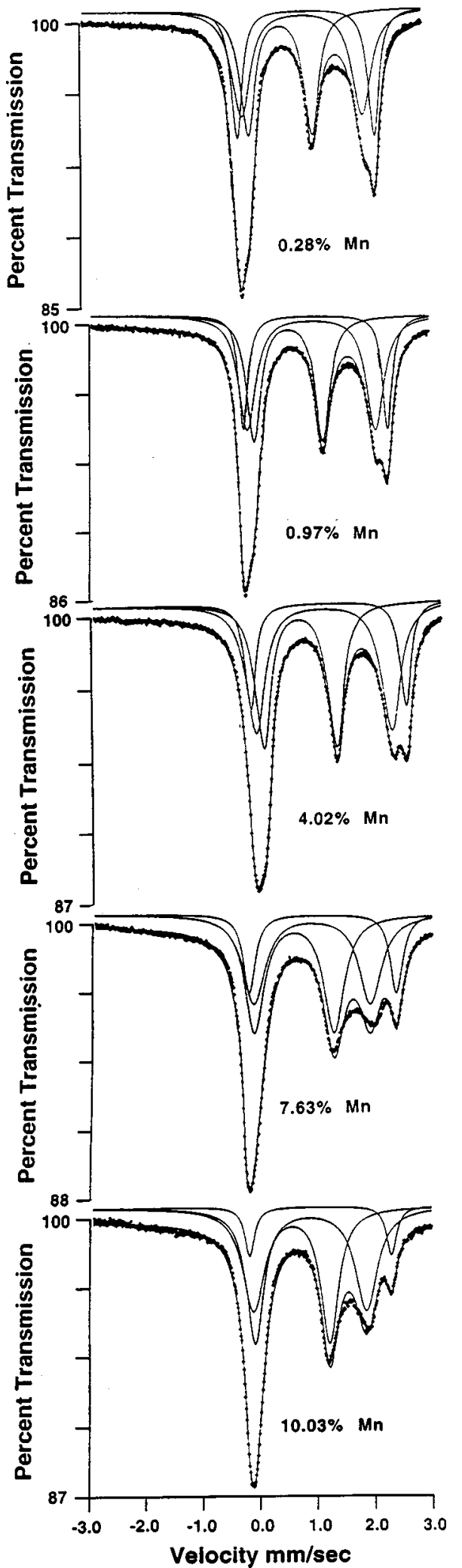

FIG. 2. ${ }^{57} \mathrm{Fe}$ Mössbauer spectra at $295 \mathrm{~K}$ of ilvaites with different $\mathrm{Mn}$ contents.

and below $50 \mathrm{~K}^{5,8,9}$ corresponding to the antiferromagnetic ordering of $\mathrm{Fe}^{2+}$ and $\mathrm{Fe}^{3+}$ ions on the $M 11$ and $M 12$ sites and $\mathrm{Fe}^{2+}$ on the $M 2$ site, respectively.

Previous magnetic susceptibility studies on two ilvaite specimens from Japanese localities having Mn contents of 0.3 and $7 \mathrm{wt} \%$ did not reveal any relationship between

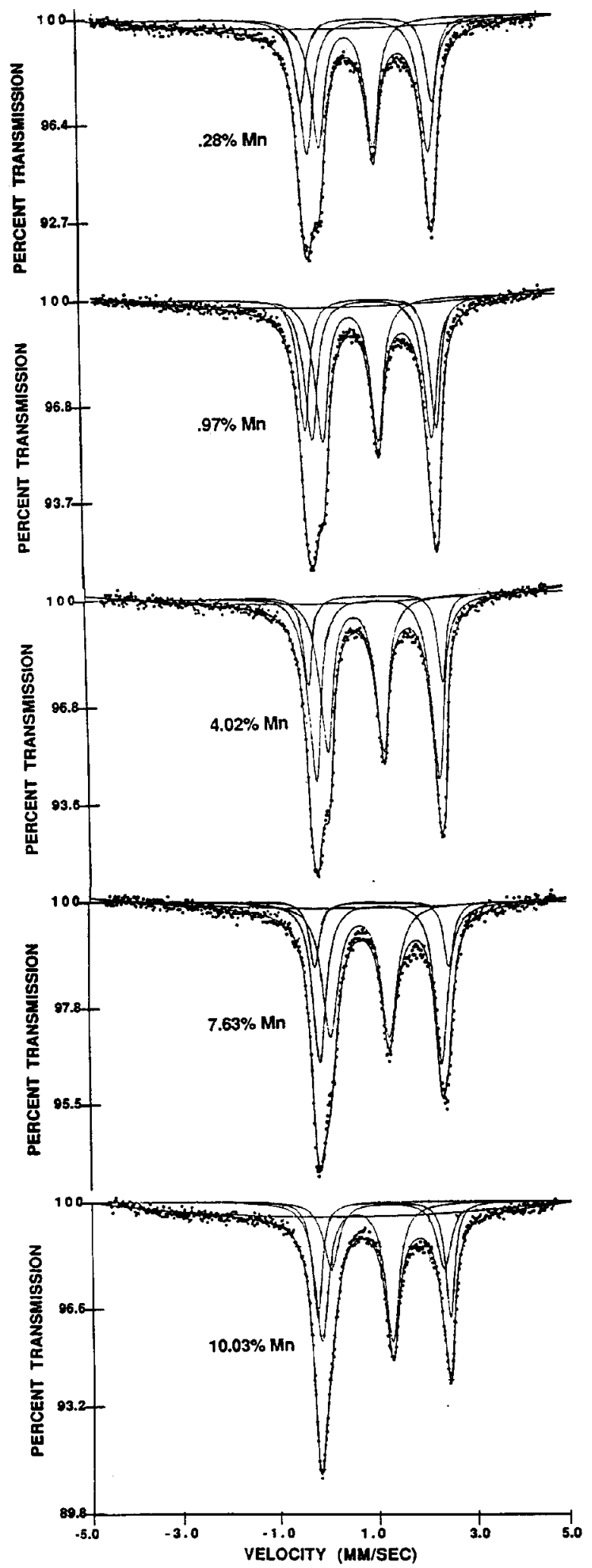

FIG. 3. ${ }^{57} \mathrm{Fe}$ Mössbauer spectra at $160 \mathrm{~K}$ of ilvaites with different $\mathrm{Mn}$ contents.

chemical composition and magnetic ordering temperatures. ${ }^{2}$ This finding is contrary to an earlier study in this laboratory on three natural samples of ilvaite having Mn contents of $0.4,1.2$, and $3.3 \mathrm{wt} \%$ in which an inverse relationship was found between $\mathrm{Mn}$ content and the magnetic ordering temperature of $\mathrm{Fe}^{2+}$ ions on the $M 2$ sites. ${ }^{9}$ 
TABLE I. ${ }^{57} \mathrm{Fe}$ Mössbauer spectroscopic parameters for ilvaite specimens collected below $160 \mathrm{~K}$.

\begin{tabular}{rlcr}
\hline \hline Sample & Site & $\Delta E_{Q}\left(\mathrm{~mm} \mathrm{~s}^{-1}\right)$ & $\delta\left(\mathrm{mm} \mathrm{s}^{-1}\right)^{\mathrm{a}}$ \\
\hline \multirow{2}{*}{1500} & $M 12$ & 1.14 & .64 \\
& $M 11$ & 2.53 & 1.09 \\
& $M 2$ & 2.75 & 1.07 \\
777 & $M 12$ & 1.17 & .64 \\
& $M 11$ & 2.50 & 1.09 \\
5022 & $M 2$ & 2.75 & 1.07 \\
& $M 12$ & 1.17 & .61 \\
& $M 11$ & 2.57 & 1.05 \\
22684 & $M 2$ & 2.80 & 1.06 \\
& $M 12$ & 1.25 & .61 \\
& $M 11$ & 2.55 & 1.04 \\
M32628 & $M 2$ & 2.8 & 1.06 \\
& $M 12$ & 1.45 & .51 \\
& $M 11$ & 2.34 & 1.16 \\
& $M 2$ & 2.75 & 1.08 \\
\hline \hline
\end{tabular}

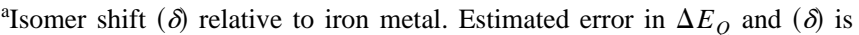
$\pm 0.01 . M 11$ and $M 12$ represent ribbon sites containing $\mathrm{Fe}^{2+}$ and $\mathrm{Fe}^{3+}$ ions. $M 2$ represents the nonribbon site containing $\mathrm{Fe}^{2+}$.

There are also ambiguities involving the influence of $\mathrm{Mn}$ content on the degree of valence fluctuation as reflected in ${ }^{57} \mathrm{Fe}$ Mössbauer spectra. Results of a study of the ${ }^{57} \mathrm{Fe}$ Mössbauer spectra at $375 \mathrm{~K}$ of synthetic samples of ilvaite indicate that the presence of $\mathrm{Mn}$ enhances the degree of valence fluctuation in ilvaite. ${ }^{10}{ }^{57} \mathrm{Fe}$ Mössbauer spectra of a series of eleven natural specimens of ilvaite, however, were interpreted as showing no relationship between Mn content and the degree of valence fluctuation., 3

Considering the ambiguities in the relationships between chemical composition and the electronic and magnetic properties of ilvaite, this study was undertaken to gain further insight into these questions using a large suite of natural ilvaite specimens. More than 43 ilvaite specimens of known provenance have been investigated by means of $x$-ray diffraction, magnetic susceptometry, ${ }^{57} \mathrm{Fe}$ Mössbauer spectroscopy, electrical conductivity, and electron microprobe analysis.

\section{EXPERIMENT}

The chemical composition of the ilvaite samples used in this study were determined using electron microprobe analysis. A complete report of the compositions and provenances of the ilvaite samples used in this study have been reported elsewhere. ${ }^{11}$ Magnetic susceptibility measurements were made using a superconducting quantum interference device (SQUID) susceptometer in a constant field of $25 \mathrm{KG}$ over a temperature range of 5-300 K. The magnetic ordering temperature of the $M 2$ site ions was extracted from plots of $1 / \chi$ vs $T$ by finding the local minimum of the function (Fig. 1). It was not possible to unambiguously extract the magnetic ordering temperature of the $M 11 / M 12$ site ions from plots of $1 / \chi$ vs $T$.

Transmission ${ }^{57} \mathrm{Fe}$ Mössbauer spectra of 46 natural ilvaite specimens were obtained at 295 and $160 \mathrm{~K}$ as described previously. ${ }^{11}$ The Mössbauer data were fitted using a least squares minimization program assuming Lorentzian line shapes, as described previously. The filled circles in the spectra represent the experimental data and the solid lines are the result of the fitting procedure (Figs. 2 and 3).

\section{RESULTS AND DISCUSSION}

The magnetic ordering temperature of the $M 2$ site ions appears as a cusp in the plot of $1 / \chi$ vs $T$ in the region below $30 \mathrm{~K}$ (Fig. 1). The extracted magnetic ordering temperatures were found to decrease with increasing Mn content. The ordering temperatures and corresponding Mn contents of the ilvaite samples studied were: $27 \mathrm{~K}-0.23 \mathrm{wt} \% \mathrm{Mn}$ (Seriphos, Greece); 26 K-0.28 wt \% Mn (Seriphos, Greece); 26 K-0.70 wt \% Mn (Seriphos, Greece); 25 K-0.90 wt \% Mn (Elba, Italy), and $23 \mathrm{~K}-0.97 \mathrm{wt} \% \mathrm{Mn}$ (Elba, Italy). The remaining samples in this suite had manganese contents of 1.20, 1.23, $2.89,3.96,4.46,4.67,7.63$, and $10.03 \mathrm{wt} \%$. None of these samples exhibited any evidence of magnetic ordering in their susceptibility data and the magnetic ordering temperatures are thought to be below $4 \mathrm{~K}$. It appears that the critical Mn content above which there is no evidence of magnetic ordering of the $M 2$ site ions from the plots of $1 / \chi$ vs $T$ is $1.2 \mathrm{wt} \%$.

Qualitative analysis of the spectral profiles of 46 Mössbauer spectra of ilvaite specimens collected at $295 \mathrm{~K}$ show changes relative to Mn content (Fig. 2). The full set of spectra and the corresponding parameters are reported elsewhere. ${ }^{11}$ In contrast to the Mössbauer spectra of the ilvaite samples collected at $295 \mathrm{~K}$ and the dependence of the features on Mn content, Mössbauer spectra of six natural ilvaite specimens collected at temperatures below $160 \mathrm{~K}$, exhibit similar spectral features (Fig. 3) and exhibit no variations in spectral features relative to Mn content. At these temperatures electron delocalization among the $M 11$ and $M 12$ sites is expected to be at a minimum. The absence of any appreciable degree of delocalization in ilvaite at these temperatures is evident in the comparison with the $295 \mathrm{~K}$ spectra. The Mössbauer parameters for the spectra collected below $160 \mathrm{~K}$ are shown in Table I.

${ }^{1}$ N. Haga and Y. Takeuchi, Z. Kristallogr. 144, 161 (1976).

${ }^{2}$ T. Yamanaka and Y. Takeuchi, Phys. Chem. Miner. 4, 149 (1979).

${ }^{3}$ D. A. Nolet and R. G. Burns, Phys. Chem. Miner. 4, 221 (1979).

${ }_{5}^{4}$ D. A. Nolet, thesis, Massachusetts Institute of Technology, 1978.

${ }^{5}$ J. M. D. Coey, N. Van Dang, and S. Ghose, J. Appl. Phys. 55, 1963 (1984).

${ }^{6}$ M. A. C. de Melo, H.-H. Klauß, F. J. Litterst, and G. Amthauer, Hyperfine Interact. 85, 145 (1994).

${ }^{7}$ R. A. Robie, H. T. Evans, Jr., and B. S. Hemingway, Phys. Chem. Miner. 15, 390 (1988).

${ }^{8}$ S. Ghose, A. W. Hewat, and M. Marezio, Phys. Chem. Miner. 11, 67 (1984).

${ }^{9}$ C. R. Bluncson and B. J. Evans, in Proceedings of the Second Nassau Conference on the Mössbauer Effect, edited by C. Wynter and E. E. Alp, (Brown, Dubuque, Iowa, 1995), pp. 81-88.

${ }^{10}$ B. Ghazi-Bayat, M. Behruz, F. J. Litterst, W. Lottermoser, and G. Amthauer, Phys. Chem. Miner. 18, 491 (1992).

${ }^{11}$ Cherie R. Dotson, thesis University of Michigan, 1998. 